\title{
Géneros televisivos preferidos y actitudes hacia la publicidad
}

Cristina Etayo-Pérez | cetayo@unav.es

UNIVERSIDAD DE NAVARRA

Resumen: Las actitudes hacia la publicidad constituyen una variable fundamental en el proceso de logro de la eficacia publicitaria. Con el fin de mejorar el conocimiento sobre ellas, en este trabajo se analizan las relaciones existentes entre las preferencias por los distintos géneros televisivos y la actitud hacia la publicidad de los consumidores. Se utilizan datos de una encuesta realizada en España y se pone de manifiesto que la preferencia por los géneros de entretenimiento está relacionada con una actitud más positiva hacia la publicidad en televisión.

Palabras clave: Publicidad, televisión, actitudes, géneros, preferencias

Abstract: Attitudes to advertising are a key variable in the effectiveness of the advertising process. The purpose of this paper is to explore the interrelationships between different television genres and consumer attitudes towards advertising. The research draws on data from a survey carried out in Spain, and discloses that viewer preferences for entertainment programs correlate with a more positive attitude towards advertising on television.

Key words: Advertising, television, attitudes, genres, preferences 


\section{Introducción}

A pesar de la creciente competencia entre medios de comunicación, la televisión continúa siendo uno de los medios preferidos tanto por consumidores como por anunciantes. De esta manera, en España, la televisión es el medio más importante en cuanto a consumo, con un promedio de 246 minutos diarios en 2012 (Kantar Media, 2013). Asimismo, ha sido el medio de comunicación que más inversión publicitaria ha venido recibiendo año tras año en términos absolutos hasta 2011, donde únicamente se ha visto superado por el mailing personalizado. Concretamente, a pesar del contexto de crisis económica generalizada, la inversión publicitaria en televisión en España durante 2012 ha ascendido a 1815, 3 millones de euros, lo que supone un $39,2 \%$ de la inversión destinada a medios convencionales y un 16,7\% del total de la inversión publicitaria en España en el año 2012. (Infoadex, 2013). Dentro del propio mercado español de la televisión, se han producido importantes cambios en lo relacionado con el reparto de las inversiones publicitarias. Desde que en enero de 2010 la televisión pública nacional eliminara la publicidad de su programación, la inversión publicitaria ha ido dirigida únicamente a televisiones comerciales.

En este contexto de transición hacia una nueva situación, en la que el modelo de negocio de los nuevos medios carece de una clara definición y, simultáneamente, los nuevos medios no convencionales son capaces de atraer grandes inversiones en publicidad, se plantea la necesidad de tener muy en cuenta el retorno de la inversión en publicidad en televisión. Aunque muchos estudios han puesto de manifiesto la importancia de la televisión como constructora de valores y de paradigmas comunes para los individuos, no puede olvidarse que lo que las empresas buscan al realizar la inversión publicitaria es una efectividad que se traduzca en un incremento en las ventas del producto anunciado. Para comprender mejor los procesos a través de los cuales se produce esta efectividad es imprescindible conocer en profundidad las actitudes de los consumidores hacia la publicidad en televisión, qué les gusta y les disgusta de ella, si la consideran de utilidad y si esas actitudes cambian con las preferencias y las características del telespectador.

Los factores asociados a las actitudes y la conducta ante la publicidad en televisión han sido profusamente estudiados desde varias perspectivas. Así, se han puesto en relación con variables macros como la situación económica general y la cultura del país y con variables sociodemográficas individuales como el sexo, la edad o el nivel socioeconómico (Roberts, 2000; Wollin, 2002; Petrovici y Marinov, 2007).

Uno de los aspectos a las que no se ha prestado demasiada atención en el estudio de las actitudes hacia la publicidad en televisión es la influencia que sobre ellas tienen los gustos del telespectador por los distintos géneros televisivos. La aportación científica de este trabajo consiste es indagar si existe relación entre ambos factores. Se analizará esta cuestión desde una perspectiva empírica. Los resultados obtenidos pueden ser de una gran relevancia para las empresas implicadas en la publicidad televisiva, como los anunciantes o los canales de 
televisión. Si existen diferencias en las actitudes del telespectador en función del tipo de programa que le gusta, es de esperar que la efectividad de la publicidad dependa del tipo de programa en el que se inserta. Dada la necesidad de optimizar los recursos dedicados a la publicidad en televisión, cada vez más amenazados por la constante aparición de nuevos medios decididos a atraer hacia sí las inversiones publicitarias, se percibe como muy necesario el análisis de la ubicación de los anuncios publicitarios dentro de la estructura de programación para lograr la máxima efectividad de estas inversiones.

Para lograr este objetivo, el presente trabajo se divide en las siguientes secciones. En primer lugar, se analizan las actitudes hacia la publicidad, identificando sus dimensiones y los factores que las determinan. Posteriormente se examina la relevancia de las preferencias por los diferentes géneros en la conducta del espectador en relación con la televisión. A continuación se describen los datos empleados en la parte empírica del trabajo, así como la forma de medición de las variables centrales. Seguidamente se explican los resultados obtenidos en el análisis empírico, para finalizar con las principales conclusiones del trabajo.

\section{Las actitudes hacia la publicidad}

La actitud hacia la publicidad se ha definido como "una predisposición aprendida a responder consistentemente de una manera favorable o desfavorable a la publicidad en general" (MacKenzie y Lutz, 1989). Esta variable es de vital importancia puesto que numerosos estudios han demostrado que la actitud favorable o desfavorable hacia la publicidad determina el comportamiento hacia la marca y la intención de compra (Lutz, 1985; Mehta, 2000; Gauzente, 2008; Dix y Phau, 2010). Por ello, también se han estudiado los distintos motivos y tipos de conductas que explican la evasión publicitaria (Shavitt, Lowery y Haefner, 1998; Tse y Lee, 2001; Van Meurs, 1998), examinando si esta conducta depende del medio a través del que se recibe el mensaje (Speck y Elliott, 1997).

A partir del trabajo inicial de Bauer y Greyser (1968) se han analizado con amplitud los factores que pueden afectar a la actitud de los consumidores hacia la publicidad. De esta manera, se ha alcanzado evidencia de que las actitudes hacia la publicidad no son homogéneas para todos los países, sino que difieren de unos a otros. Así, se ha destacado la importancia de las diferencias culturales para explicar la variedad nacional en estas actitudes (Durvasula et al. 1993; Martín-Santana y Beerli-Palacio, 2008; Nan, 2006; Ramaprasad y Thurwanger, 1998; Rojas-Méndez et al, 2009). A su vez, se ha descubierto que también hay características personales relacionadas con factores psicológicos que influyen en la actitud hacia la publicidad (Dutta-Bergman, 2006; Roberts y Manolis, 2000; Brett et al., 2009; Alwitt y Prabhaker, 1994; Andrews et al., 1994; Chan et al., 2007).

El trabajo de referencia de Pollay y Mittal (1993) estableció las principales dimensiones de las que depende la satisfacción que los televidentes obtienen de la publicidad que ven en televisión. Según estos autores, las actitudes hacia la publicidad vienen determinadas por 
dos grandes categorías de factores: unos relacionadas con la utilidad personal y otros con factores socioeconómicos. Los usos personales incluyen la publicidad como fuente de información sobre productos (Norris, 1984), como reflejo de un rol social y de una imagen (Richins, 1991), así como fuente de placer y entretenimiento. Entre los efectos sociales que se mencionan se encuentran la creencia de que la publicidad es buena para la economía, que promueve el materialismo y valores corruptos, y que conlleva afirmaciones falsas y carentes de sentido. Los diferentes sentidos y significados que los individuos atribuyen a esos factores determinarán su actitud global hacia la publicidad, esto es, si creen que es buena o mala o si les gusta o no.

\section{Las preferencias de género televisivo y las actitudes hacia la publicidad}

Dentro de la programación de televisión podemos encontrar la existencia de diferentes tipos de géneros. El género televisivo se define como una estructura que se halla en un plano superior al de las obras individuales y que está conformada por diversos elementos (Neale, 1980). Por una parte, influye en la dinámica de producción industrial (industria/autor) y en su producto (obra) y, por otra, representa un código de reconocimiento e interpretación de las obras para el espectador (Lacey, 2000). Además, el género desempeña la función de etiqueta de la industria para ofrecer los productos a sus públicos, de modo que permite a la audiencia, además de interpretar la obra, elegirla o no para su consumo (Altman, 2000). En consecuencia, estas funciones suponen un nexo entre la oferta de la industria audiovisual y los gustos del público, tanto desde el punto de vista de la comprensión como del consumo. En este sentido puede decirse que los géneros facilitan la elección de consumo por parte de los espectadores y que sólo existen si son reconocidos como tales por la crítica y el público (Aumont, 2006).

Recientemente ha habido algunos intentos de inferir modelos que faciliten el análisis de los géneros televisivos, aunque aplicados a programas concretos, e incluso se han presentado propuestas metodológicas para medir la diversidad de los programas emitidos por televisión (Hellman, 2001). En este contexto se ha prestado más atención al tema de si existen diferencias en las preferencias que las personas muestran por unos géneros y por otros y al análisis de las variables que pueden influir en estas preferencias.

En la medida en que las preferencias por los distintos géneros constituyen un rasgo identificativo de las actitudes del individuo hacia la televisión, consideramos que van a ser un factor que contribuye a determinar las opiniones del individuo hacia la publicidad en televisión. Para profundizar en esta relación emplearemos una clasificación de los géneros televisivos en cuatro categorías: entretenimiento, ficción, informativos y deportes. Se trata de una clasficación que acoge todo tipo de contenidos televisivos y que da lugar a una tipología de géneros con características muy distintas, de tal manera que podemos esperar que las preferencias por unos $u$ otros puedan influir en las actitudes del telespectador hacia la publicidad. 
Así, por ejemplo el tipo de formato que presentan los programas de entretenimiento tiene ciertas similitudes con el formato publicitario que otros géneros no presentan. Puede decirse que entre todos los géneros televisivos, el de los programas de entretenimiento es el que en mayor medida se asemeja al formato de la publicidad. Ambos tienden a combinar tramas cortas que dependen del suspense y de secuencias animadas que exigen mucha atención durante cortos periodos de tiempo, antes de relajar el ritmo narrativo. Ninguno de los dos formatos supone una gran cantidad de pensamiento consciente ya que las historias en ambas casos se presentan como dadas y no requieren de un mayor desarrollo por parte de la audiencia. El ritmo de ambos formatos es rápido y discontinuo, de manera que si se pierde una parte, no se tienen dificultades en retomar después el hilo narrativo. Podría esperarse pues que una mayor preferencia por este tipo de contenidos esté relacionada con una actitud hacia la publicidad mas positiva.

Otro tema analizado en la actitud hacia la publicidad es el intrusismo. Las personas que perciben la publicidad como intrusiva muestran una peor actitud hacia ella. En este sentido, los contenidos informativos, de ficción o deportivos requieren una continuidad mayor de la que puede necesitar un programa de entretenimiento. En consecuencia, puede esperarse que los espectadores a los que les gusten los informativos, la ficción o los programas de deportes consideren a la publicidad más intrusiva que aquellos que prefieren contenidos de entretenimiento.

Finalmente, no debemos olvidar que una de las dimensiones que conforman la actitud hacia la publicidad es su papel como fuente informativa sobre el producto. Desde esta perspectiva, podria esperarse una relación entre la preferencia por los programas informativos y una mejor valoración de esta dimensión de la publicidad.

\section{Datos y metodologia}

\subsection{Muestra}

Los datos empleados en la parte empírica del trabajo fueron recogidos mediante una encuesta, en la que la población objetivo estaba formada por todos los residentes en España de al menos 14 años de edad (37.910.000 habitantes). La selección muestral fue polietápica, estratificada por región (centro, sur, norte y este) y tamaño del municipio $(<5.000$ habitantes, 5.000-50.000, más de 50.000 y capitales de provincia). El tamaño de la muestra es 1.000 personas. Para un nivel de confianza del $95 \%$ esto implica un margen de error del $3.2 \%$. Las entrevistas fueron realizadas telefónicamente asistidas por ordenador (CATI). La selección del entrevistado en cada hogar se realizó con cuotas de género y edad.

Para controlar los posibles efectos de varianza común debido al método de recogida de la información, se tuvieron en cuenta varias recomendaciones mencionadas en la literatura 
(Podsakoff et al., 2003). Por ejemplo, se emplearon diferentes formatos de respuesta para la medición de las variables. Además, los ítems empleados se basaron en escalas ampliamente utilizadas y contrastadas en la literatura. El pre-test realizado sirvió para asegurarnos de que la formulación de los ítems no era ambigua, así como de que éstos eran fácilmente entendibles. Para reducir las posibles reticencias a responder de los entrevistados y que éstos tendieran en menor medida a ofrecer respuestas socialmente aceptables, se garantizó completamente el anonimato. También se realizó el test de un factor de Harman. Dado que la solución del análisis factorial sin rotación de las dieciocho variables finalmente utilizadas en el estudio dio lugar a siete factores con valores propios mayores que uno, explicando el primer factor únicamente el 16 por ciento de la varianza común, se puede concluir que los resultados aquí presentados no están afectados por problemas derivados de la varianza común debida al método.

\subsection{Variables}

\subsubsection{Variables dependientes}

Las creencias y actitudes hacia la publicidad en televisión se miden a partir del modelo propuesto por Pollay y Mittal (1993). Por tanto, los siguientes constructos reflectivos han sido considerados: información sobre el producto, imagen social, hedonismo, corrupción de valores, veracidad, materialismo, beneficio para la economía, intrusismo y actitudes generales hacia la publicidad en televisión.

Como es habitual en la literatura, estos constructos se midieron utilizando indicadores reflectivos. Exceptuando aquellos relacionados con las actitudes generales hacia la publicidad en televisión, los ítems originales se formularon en términos de acuerdo/desacuerdo en una escala de uno a cinco. Las medias y desviaciones típicas se muestran en la Tabla 1. El alfa de Cronbach para todos los constructos excepto uno excede el coeficiente alfa mínimo exigido de 0,60, tal y como recomienda Nunally (1978). La excepción es el hedonismo. No obstante, el valor del alfa de Cronbach para este constructo es de 0,598, muy próximo al 0,60 habitualmente requerido.

Tabla 1. Estadísticos descriptivos para las medidas de creencias y actitudes hacia la televisión

\begin{tabular}{|c|c|c|c|}
\hline & Media & $\begin{array}{l}\text { Desv. } \\
\text { Típ. }\end{array}$ & $\begin{array}{l}\text { Alfa de } \\
\text { Cronbach }\end{array}$ \\
\hline Información & 3,107 & 0,817 & \multirow[t]{4}{*}{0,656} \\
\hline $\begin{array}{l}\text { Es una fuente valiosa de información sobre los } \\
\text { productos }\end{array}$ & 3,120 & 1,086 & \\
\hline Dice qué marcas tienen las características que busco & 2,797 & 1,070 & \\
\hline Me ayuda a estar al día de lo que hay en el mercado & 3,400 & 1,015 & \\
\hline Imagen social & 2,507 & 0,833 & \multirow[t]{4}{*}{0,707} \\
\hline A través de ella sé qué está de moda & 2,767 & 1,136 & \\
\hline Me dice qué compra y usa la gente como yo & 2,549 & 1,024 & \\
\hline Me ayudan a conocer qué productos reflejan cómo & 2,200 & 0,921 & \\
\hline
\end{tabular}




\begin{tabular}{|c|c|c|c|}
\hline soy & & & \multirow{5}{*}{0,598} \\
\hline Hedonismo & 2,791 & 0,829 & \\
\hline A menudo es entretenida y divertida & 2,928 & 1,117 & \\
\hline Me divierto recordando anuncios & 3,229 & 1,108 & \\
\hline $\begin{array}{l}\text { A veces disfruto más con los anuncios que con los } \\
\text { programas }\end{array}$ & 2,227 & 1,084 & \\
\hline Corruptora de valores & 3,663 & 0,777 & \multirow[t]{4}{*}{0,628} \\
\hline Promueve valores no deseables & 3,560 & 1,020 & \\
\hline Se aprovecha de los niños & 3,885 & 0,968 & \\
\hline Hace que la gente viva en un mundo de fantasía & 3,575 & 1,059 & \\
\hline Veracidad & 2,388 & 0,856 & \multirow[t]{3}{*}{0,620} \\
\hline Presenta una imagen verdadera de lo anunciado & 2,341 & 0,983 & \\
\hline Lo anunciado cumple con las promesas realizadas & 2,439 & 0,987 & \\
\hline Materialismo & 4,096 & 0,685 & \multirow[t]{5}{*}{0,722} \\
\hline $\begin{array}{l}\text { Hace que somos estemos interesados en comprar y } \\
\text { poseer }\end{array}$ & 4,212 & 0,923 & \\
\hline $\begin{array}{l}\text { Hace que la gente compre cosas caras sólo para } \\
\text { aparentar }\end{array}$ & 4,079 & 0,940 & \\
\hline Hace que la gente compre cosas que no necesita & 4,242 & 0,807 & \\
\hline $\begin{array}{l}\text { Frustra porque muestra productos que la gente no } \\
\text { puede comprar }\end{array}$ & 3,850 & 1,023 & \\
\hline Buena para la economía & 2,667 & 0,809 & \multirow[t]{4}{*}{0,647} \\
\hline Ayuda a mejorar el nivel de vida de la sociedad & 2,212 & 0,903 & \\
\hline Da lugar a mejores productos para el público & 2,663 & 1,048 & \\
\hline $\begin{array}{l}\text { Promueve la competencia, lo que beneficia al } \\
\text { consumidor }\end{array}$ & 3,133 & 1,154 & \\
\hline Intrusismo & 4,473 & 0,638 & \multirow[t]{4}{*}{0,706} \\
\hline $\begin{array}{llll}\text { Los cortes publicitarios contienen } & \text { demasiados } \\
\text { anuncios }\end{array}$ & 4,537 & 0,799 & \\
\hline Se repiten mucho los mismos anuncios & 4,400 & 0,829 & \\
\hline $\begin{array}{l}\text { La publicidad aparece siempre en el momento más } \\
\text { inoportuno }\end{array}$ & 4,486 & 0,795 & \\
\hline Actitudes generales & 2,669 & 0,896 & \multirow[t]{5}{*}{0,849} \\
\hline Muy buena / muy mala & 2,771 & 1,118 & \\
\hline Me gusta / me disgusta & 2,438 & 1,114 & \\
\hline Muy favorable / muy desfavorable & 2,745 & 1,042 & \\
\hline Muy positiva / muy negativa & 2,724 & 1,051 & \\
\hline
\end{tabular}

\subsubsection{Variables independientes}

Se emplearon cuatro índices para medir las preferencias de los espectadores en relación con los diferentes géneros televisivos. Cada índice recoge los gustos del telespectador en lo que se refiere a los siguientes tipos de programas: entretenimiento, ficción, informativos y deportes. El entrevistado debía expresar en una escala de 1 (nada) a 5 (muchísimo) lo que le gustaban los siguientes géneros televisivos: noticias, magazines y talk shows, películas, series, concursos, programas deportivos, documentales, telerrealidad, musicales, entretenimiento y humor, y divulgativos.

Los índices se crearon asignando a cada uno de estos géneros una de las cuatro grandes categorías: entretenimiento incluye magazines y talk shows, concursos, telerrealidad, musicales, y entretenimiento y humor; ficción comprende películas y series; informativos incluye noticias, documentales y divulgativos; y deportes solamente incluye los programas deportivos. El valor del índice se calculó como el promedio de los ítems que lo formaban. 
Al contrario que en la medición de las variables dependientes, las variables independientes empleadas para medir las preferencias por los diferentes géneros televisivos se basan en el uso de indicadores formativos. Dada la naturaleza de los conceptos implicados, el uso de este tipo de medidas se considera apropiado ya que las medidas formativas suponen que las medidas influyen conjuntamente sobre la variable latente y que el significado emana de las medidas de la variable, en el sentido de que el sentido completo de la variable compuesta latente se deriva de sus medidas (Mackenzie et al., 2005). En las medidas formativas, la variable latente viene determinada por sus indicadores y no a la inversa.

La naturaleza de los constructos latentes formativos hace que la perspectiva de consistencia interna resulte inapropiada para valorar su adecuación (Bagozzi, 1994). En nuestro caso hemos estudiado la multicolinealidad de las medidas de un mismo índice con el fin de validar los constructos formativos (Podzakoff et al., 2006). En este contexto, un Factor de Inflación de la Varianza por debajo de cinco es un buen indicador de ausencia de multicolinealidad (Judge et al., 1988). Como figura en la Tabla 2, los valores del Factor de Inflación de la Varianza (FIV) para los ítems empleados en las variables de preferencia de géneros cumplen con este criterio.

Tabla 2. Estadísticos descriptivos para las medidas de preferencia de géneros

\begin{tabular}{|l|c|c|c|}
\hline & Media & $\begin{array}{c}\text { Desviación } \\
\text { típica }\end{array}$ & FIV \\
\hline Entretenimiento & 2,281 & 0,837 & \\
\hline Magazine y talk shows & 1,787 & 1,259 & 1,177 \\
\hline Concursos & 2,516 & 1,369 & 1,211 \\
\hline Telerrealidad & 1,655 & 1,159 & 1,250 \\
\hline Musicales & 2,592 & 1,420 & 1,164 \\
\hline Entretenimiento y humor & 2,853 & 1,460 & 1,120 \\
\hline Ficción & 3,547 & 1,076 & 1,075 \\
\hline Películas & 3,863 & 1,245 & 1,075 \\
\hline Series & 3,222 & 1,425 & 1,070 \\
\hline Informativos & 3,266 & 0,892 & 1,078 \\
\hline Noticias & 3,910 & 1,108 & 1,045 \\
\hline Documentales & 3,484 & 1,360 & \\
\hline Divulgativos & 2,395 & 1,451 & \\
\hline Deportes & 2,791 & 1,616 & \\
\hline
\end{tabular}

\subsubsection{Variables de control}

El género, la edad y la educación se emplearon como variables de control en nuestros análisis. Para medir la educación se emplearon tres variables ficticias para los estudios primarios, los estudios secundarios y los universitarios, siendo la categoría omitida sin estudios.

La media, la desviación típica y la matriz de correlaciones para las variables dependientes, independientes y de control figura en la Tabla 3. 
4.3. Método de estimación

El método empleado en las estimaciones es la regresión por mínimos cuadrados ordinarios. Para cada variable dependiente se estimó un modelo que incluyera las variables de control. 
Tabla 3. Estadísticos descriptivos y matriz de correlaciones para las variables dependientes, independientes y de control

\begin{tabular}{|c|c|c|c|c|c|c|c|c|c|c|c|c|c|c|c|c|c|c|c|}
\hline & Media & D.t. & 1 & 2 & 3 & 4 & 5 & 6 & 7 & 8 & 9 & 10 & 11 & 12 & 13 & 14 & 15 & 16 & 17 \\
\hline 1. Hombre & 0,47 & 0,49 & & & & & & & & & & & & & & & & & \\
\hline 2. Edad & 46,43 & 18,49 &,- 04 & & & & & & & & & & & & & & & & \\
\hline 3. Primarios & 0,26 & 0,44 & 00 &, $16^{* * * *}$ & & & & & & & & & & & & & & & \\
\hline 4. Secundarios & 0,39 & 0,48 & $\begin{array}{l}-, 03 \\
\end{array}$ & $\begin{array}{c}- \\
-22^{* * *} \\
\end{array}$ & $\begin{array}{c}- \\
, 48 * * \\
\end{array}$ & & & & & & & & & & & & & & \\
\hline 5. Universitarios & 0,28 & 0,45 &, $07^{* * *}$ &,$- 06^{* *}$ &, $37 * *$ & $\begin{array}{c}- \\
, 50^{* * *}\end{array}$ & & & & & & & & & & & & & \\
\hline 6. Entretenimiento & 2,28 & 0,83 & $\begin{array}{c}- \\
15^{* * *} \\
\end{array}$ &,- 04 &, $22 * * *$ &,$- 06^{*}$ & $\begin{array}{c}- \\
, 23^{* * *} \\
\end{array}$ & & & & & & & & & & & & \\
\hline 7. Ficción & 3,54 & 1,07 & $\begin{array}{c}- \\
09^{* * *}\end{array}$ & $\begin{array}{c}- \\
-34^{* * *}\end{array}$ &,- 04 &, $08^{* * *}$ &,- 02 &, $29 * * *$ & & & & & & & & & & & \\
\hline 8. Informativos & 3,26 & 0,89 &,- 04 & $39 * * *$ &, $07 * *$ &,$- 05^{*}$ &,$- 06 * *$ &, $13 * * *$ &,- 02 & & & & & & & & & & \\
\hline 9. Deportes & 2,79 & 1,61 & $46^{* * *}$ & $\begin{array}{c}- \\
-08^{* * *}\end{array}$ & 02 & 01 &,- 02 & 01 & 0,00 &,- 02 & & & & & & & & & \\
\hline $\begin{array}{ll}\text { 10. Información } & \text { del } \\
\text { producto } & \\
\end{array}$ & 3,10 & 0,81 &, 00 &,- 04 &, $05^{*}$ &, 00 &,$- 07 * *$ &, $24 * * *$ &, $06^{*}$ &, 01 & ,05 & & & & & & & & \\
\hline 11. Imagen social & 2,50 & 0,83 & ,02 &,- 04 &, $09 * * *$ &,- 02 &,$- 08^{* *}$ &, $26 * * *$ &, $07 * *$ &,- 02 &, $06^{* *}$ &, $47 * * *$ & & & & & & & \\
\hline 12. Hedonismo & 2,79 & 0,82 &,- 01 &,- 04 & 0,00 &, 02 &,- 02 &, $15^{* * *}$ &, $09^{* * * *}$ &, $057^{*}$ &, $06^{*}$ &, $25 * * *$ &, $22 * * *$ & & & & & & \\
\hline 13. Corruptora de valores & 3,66 & 0,77 &,- 01 &, $12 * * *$ & ,01 &,- 02 & 00 & $\begin{array}{c}- \\
-13^{* * *}\end{array}$ &,$- 06 * *$ &, $07^{* *}$ & $\begin{array}{c}- \\
, 06^{* *} \\
\end{array}$ & $\begin{array}{c}- \\
, 14^{* * *} \\
\end{array}$ &,$- 07 * *$ & $\begin{array}{c}- \\
, 11^{* * *}\end{array}$ & & & & & \\
\hline 14. Falsedad & 2,38 & 0,85 & , 00 &, $08 * * *$ &, $09 * * *$ &,$- 06 * *$ & $\begin{array}{c}- \\
, 08^{* * *}\end{array}$ &, $12 * * *$ &,- 04 & ,00 &, 04 &, $31 * * *$ &, $27 * * *$ &, $24 * * *$ & $\begin{array}{c}- \\
, 17^{* * *}\end{array}$ & & & & \\
\hline 15. Materialismo & 4,09 & 0,68 & ,00 &, $06^{* *}$ &,$- 06 * *$ & ,03 & ,03 & $\begin{array}{c}- \\
, 09^{* * *}\end{array}$ &,- 01 &, $06^{* *}$ & $-\overline{-}, 06^{* *}$ &, $05^{*}$ &,- 03 & $\begin{array}{c}- \\
-08^{* * *} \\
\end{array}$ &, $34 * * *$ & $\begin{array}{c}- \\
, 19^{* * *}\end{array}$ & & & \\
\hline $\begin{array}{llll}\begin{array}{l}\text { 16. Buena } \\
\text { economía }\end{array} & \text { para } & \text { la } \\
\end{array}$ & 2,66 & 0,80 &,- 01 & ,03 &, $11 * * *$ &,- 02 & $\begin{array}{c}- \\
, 12 * * * \\
\end{array}$ &, $24 * * *$ & ,04 & ,06* &, $07^{* *}$ &, $49 * * *$ &, $41 * * *$ &, $22 * * *$ & $\begin{array}{c}- \\
, 19 * * * \\
\end{array}$ &, $29 * * *$ & $\begin{array}{c}- \\
, 12 * * * \\
\end{array}$ & & \\
\hline 17. Intrusismo & 4,47 & 0,63 & ,01 &, $05^{*}$ &, 00 & ,01 &,- 02 &,- 04 & ,04 & $11 * * *$ & ,01 & $\begin{array}{c}- \\
-08^{* * *}\end{array}$ &,$- 06^{*}$ & $\begin{array}{c}- \\
-08^{* * *}\end{array}$ &, $19 * * *$ & $\begin{array}{c}- \\
, 16^{* * *}\end{array}$ & $22 * * *$ &,$- 07 * *$ & \\
\hline 18. Actitud general & 2,66 & 0,89 & $\begin{array}{c}- \\
08^{* * *}\end{array}$ &,$- 06 * *$ & ,01 &, 00 & ,00 &, $22 * * *$ &, $07^{* *}$ & ,02 & ,02 & $23 * * *$ &, $14 * * *$ & $41 * * *$ & $\begin{array}{c}- \\
17^{* * * *}\end{array}$ &, $23 * * *$ & $\begin{array}{c}- \\
, 09 * * *\end{array}$ &, $24 * * *$ & $\frac{-}{20^{* * *}}$ \\
\hline
\end{tabular}




\section{Resultados}

La Tabla 4 presenta los resultados del análisis de regresión llevado a cabo para examinar el papel de las preferencias de géneros televisivos a la hora de explicar las actitudes y creencias de los telespectadores españoles respecto a la publicidad en televisión.

Se ha realizado un análisis consistente en estimar un modelo para cada una de las dimensiones que, según el modelo de Pollay y Mittal, afectan a las actitudes de los espectadores hacia la publicidad en televisión. Para cada una de estos factores se plantean como variables explicativas, además de la preferencia por los distintos géneros de televisión (entretenimiento, ficción, informativos, y deportes), las variables sexo, edad, y nivel de estudios.

En la primera estimación se puede observar cómo las preferencias por los programas de entretenimiento son las únicas que afectan a la percepción de la publicidad como proveedora de información sobre los productos anunciados. De este modo, aquellos televidentes a los que les gustan los programas de entretenimiento son los únicos que utilizan la publicidad como fuente de información sobre los productos. Aunque, en principio cabría esperar una relación entre la preferencia por los programas informativos y una mejor valoración de esta dimensión de la publicidad, los resultados nos muestran que esta relación no existe.

Lo mismo ocurre con el grado de creencia en la publicidad en televisión como reflejo de la imagen social. También en este caso aquellos consumidores que tienen especial preferencia por los programas televisivos de entretenimiento son los que consideran que la publicidad les ayuda a construir su propia imagen social.

En lo que se refiere a la explicación del carácter hedónico de la publicidad, surgen con carácter significativo otras preferencias televisivas distintas de las del entretenimiento. Así, aquellos telespectadores a los que les gustan los programas informativos o los deportivos, disfrutan viendo la publicidad en TV. No obstante, son aquellos que prefieren los programas de entretenimiento los que en mayor medida se divierten con los anuncios que se emiten.

Pasando a analizar los determinantes de las creencias negativas nos encontramos con que cuanto menor es la preferencia por los programas de entretenimiento mayor es la consideración de la publicidad como un canal a través del cual se transmiten valores inadecuados. También se observa que la preferencia por los programas televisivos de carácter informativo va unida a una percepción negativa dentro de este ámbito de la publicidad en televisión. Se observa en este punto una diferencia sustancial entre el tipo de personas a las que les gustan los informativos y aquellos que prefieren los programas de entretenimiento. Estos últimos no consideran que la publicidad sea corruptora de valores a diferencia de los primeros. 
La veracidad implícita en la publicidad es la única dimensión de entre las creencias y actitudes analizadas para la que no se ha detectado ninguna influencia significativa de las preferencias de género televisivo. Es decir, el preferir un tipo de programas a otros no condiciona el que la publicidad se perciba como veraz. En este caso la única variable influyente es el nivel de estudios, en el sentido de que las personas con mayor nivel de formación dudan de la veracidad de los anuncios emitidos en televisión más que aquellos con más bajo nivel de estudios

Para la concepción de la publicidad en televisión como algo que fomenta el materialismo en la sociedad los resultados muestran que la mayor inclinación por los programas de entretenimiento conduce a una menor valoración de la publicidad en televisión como materialista. Análogo resultado se consigue para el gusto por los deportes en televisión. Por el contrario, la atracción por los programas de corte informativo se encuentra asociada a una mayor evaluación de la publicidad como portadora de valores materialistas. Dicho de otro modo, los aficionados a programas de entretenimiento y a los deportes creen que la publicidad no fomenta el materialismo, mientras que aquellos que prefieren informativos creen que sí.

En lo que respecta a la creencia de que la publicidad en TV es buena para la economía del país surgen como significativas las variables que recogen el gusto por los programas de entretenimiento y de deportes. Son los que muestran preferencia por estos programas los que creen que la publicida es buena para la economía, por su influencia positiva en el consumo, y por lo tanto en el PIB.

El intrusismo percibido de la publicidad también se ve significativamente afectado por los gustos televisivos. Cuanto menos se prefieren los programas de entretenimiento, más se considera que la publicidad en TV desempeña un papel intrusivo. Por el contrario, los telespectadores a los que les gustan la ficción y los programas divulgativos tienden en mayor medida a considerar que la publicidad interfiere negativamente en su actividad de visionado televisivo. Las distintas características personales de los telespectadores podrían explicar este hecho. Se ha encontrado relación entre personalidad extravertida y preferencia por programas de entretenimiento (Shim y Paul, 2007). Pudiera ser que los individuos con este tipo de personalidad se sientan más cómodos con una programación que incluye continuos cambios de ritmo que frente a una estructura más lineal. El formato de los espacios publicitarios, sin continuidad temática y con un ritmo rápido puede satisfacer más a aquellos que presentan esteste tipo de personalidad. Finalmente, el análisis de las actitudes generales hacia la publicidad presenta resultados en consonancia con los obtenidos para las diferentes creencias: la inclinación hacia el género del entretenimiento influye positivamente en la visión de conjunto positiva de la publicidad en TV. Sin embargo, en relación con el resto de géneros no se detecta ninguna asociación relevante. Esta actitud general hacia la publicidad se ve también afectada por la varialbe sexo. Los resutados muestran que la 
actitud general de los hombres hacia la publicidad es peor que la de las mujeres. No se observan efectos relacionados con la edad en ninguna de las varaibles explicadas. 
Tabla 4. Resultados de las regresiones

\begin{tabular}{|c|c|c|c|c|c|c|c|c|c|}
\hline & $\begin{array}{l}\text { Información } \\
\text { del producto }\end{array}$ & $\begin{array}{c}\text { Imagen } \\
\text { social }\end{array}$ & Hedonismo & $\begin{array}{l}\text { Corruptora } \\
\text { de valores }\end{array}$ & Veracidad & Materialismo & $\begin{array}{c}\text { Buena } \\
\text { para la } \\
\text { economía }\end{array}$ & Intrusismo & $\begin{array}{l}\text { Actitud } \\
\text { general }\end{array}$ \\
\hline Constante & $\begin{array}{c}2,724 * * * \\
(0,215)\end{array}$ & $\begin{array}{c}2,021 * * * \\
(0,216)\end{array}$ & $\begin{array}{c}2,158 * * * \\
(0,220)\end{array}$ & $\begin{array}{c}3,763 * * * \\
(0,206)\end{array}$ & $\begin{array}{c}2,534 * * * \\
(0,229)\end{array}$ & $\begin{array}{c}3,895 * * * \\
(0,180)\end{array}$ & $\begin{array}{c}2,168 * * * \\
(0,210)\end{array}$ & $\begin{array}{c}4,141 * * * \\
(0,170)\end{array}$ & $\begin{array}{c}2,126 * * * \\
(0,232)\end{array}$ \\
\hline Hombre & $\begin{array}{c}0,038 \\
(0,059)\end{array}$ & $\begin{array}{c}0,088 \\
(0,059)\end{array}$ & $\begin{array}{l}-0,023 \\
(0,060)\end{array}$ & $\begin{array}{l}-0,004 \\
(0,057)\end{array}$ & $\begin{array}{c}0,005 \\
(0,063)\end{array}$ & $\begin{array}{c}0,036 \\
(0,050)\end{array}$ & $\begin{array}{l}-0,015 \\
(0,058)\end{array}$ & $\begin{array}{c}0,006 \\
(0,047)\end{array}$ & $\begin{array}{c}-0,136 * * \\
(0,064)\end{array}$ \\
\hline Edad & $\begin{array}{l}-0,002 \\
(0,002)\end{array}$ & $\begin{array}{l}-0,001 \\
(0,002)\end{array}$ & $\begin{array}{l}-0,002 \\
(0,002)\end{array}$ & $\begin{array}{l}0,003 * \\
(0,002)\end{array}$ & $\begin{array}{c}0,002 \\
(0,002)\end{array}$ & $\begin{array}{l}0,003 * \\
(0,001)\end{array}$ & $\begin{array}{c}0,001 \\
(0,002)\end{array}$ & $\begin{array}{c}0,001 \\
(0,001)\end{array}$ & $\begin{array}{l}-0,003 \\
(0,002)\end{array}$ \\
\hline $\begin{array}{l}\text { Estudios } \\
\text { primarios }\end{array}$ & $\begin{array}{c}-0,074 \\
(0,120)\end{array}$ & $\begin{array}{c}0,010 \\
(0,121)\end{array}$ & $\begin{array}{c}-0,053 \\
(0,121)\end{array}$ & $\begin{array}{c}-0,029 \\
(0,114)\end{array}$ & $\begin{array}{l}-0,237^{*} \\
(0,128) \\
\end{array}$ & $\begin{array}{c}0,032 \\
(0,100)\end{array}$ & $\begin{array}{l}-0,058 \\
(0,116)\end{array}$ & $\begin{array}{l}-0,007 \\
(0,094)\end{array}$ & $\begin{array}{c}0,019 \\
(0,128)\end{array}$ \\
\hline $\begin{array}{l}\text { Estudios } \\
\text { secundarios }\end{array}$ & $\begin{array}{c}-0,072 \\
(0,123)\end{array}$ & $\begin{array}{c}-0,049 \\
(0,124)\end{array}$ & $\begin{array}{c}0,013 \\
(0,124)\end{array}$ & $\begin{array}{c}-0,067 \\
(0,116)\end{array}$ & $\begin{array}{c}-0,378 * * * \\
(0,131) \\
\end{array}$ & $\begin{array}{c}0,131 \\
(0,102)\end{array}$ & $\begin{array}{c}-0,148 \\
(0,119)\end{array}$ & $\begin{array}{c}-0,018 \\
(0,096)\end{array}$ & $\begin{array}{c}0,067 \\
(0,131)\end{array}$ \\
\hline $\begin{array}{l}\text { Estudios } \\
\text { universitarios }\end{array}$ & $\begin{array}{l}-0,106 \\
(0,125)\end{array}$ & $\begin{array}{c}-0,074 \\
(0,127)\end{array}$ & $\begin{array}{c}0,010 \\
(0,127)\end{array}$ & $\begin{array}{c}-0,093 \\
(0,119)\end{array}$ & $\begin{array}{c}-0,409 * * * \\
(0,134)\end{array}$ & $\begin{array}{c}0,115 \\
(0,105) \\
\end{array}$ & $\begin{array}{l}-0,235^{*} \\
(0,122)\end{array}$ & $\begin{array}{l}-0,065 \\
(0,098)\end{array}$ & $\begin{array}{c}0,147 \\
(0,134)\end{array}$ \\
\hline Entretenimiento & $\begin{array}{c}0,236 * * * \\
(0,035) \\
\end{array}$ & $\begin{array}{c}0,268 * * * \\
(0,035)\end{array}$ & $\begin{array}{c}0,130 * * * \\
(0,036) \\
\end{array}$ & $\begin{array}{c}-0,129 * * * \\
(0,033)\end{array}$ & $\begin{array}{c}0,105 \\
(0,037) \\
\end{array}$ & $\begin{array}{c}-0,067 * * \\
(0,029)\end{array}$ & $\begin{array}{c}0,208 * * * \\
(0,034)\end{array}$ & $\begin{array}{c}-0,069 * * \\
(0,028)\end{array}$ & $\begin{array}{c}0,246 * * * \\
(0,038)\end{array}$ \\
\hline Ficción & $\begin{array}{c}-0,014 \\
(0,027)\end{array}$ & $\begin{array}{c}0,001 \\
(0,027)\end{array}$ & $\begin{array}{c}0,038 \\
(0,027)\end{array}$ & $\begin{array}{c}0,005 \\
(0,026)\end{array}$ & $\begin{array}{c}-0,033 \\
(0,029) \\
\end{array}$ & $\begin{array}{c}0,017 \\
(0,023)\end{array}$ & $\begin{array}{c}-0,006 \\
(0,026)\end{array}$ & $\begin{array}{c}0,049 * * \\
(0,021)\end{array}$ & $\begin{array}{c}-0,013 \\
(0,029)\end{array}$ \\
\hline Informativos & $\begin{array}{c}0,001 \\
(0,032)\end{array}$ & $\begin{array}{c}-0,046 \\
(0,032)\end{array}$ & $\begin{array}{c}0,058^{*} \\
(0,033)\end{array}$ & $\begin{array}{c}0,051^{*} \\
(0,030)\end{array}$ & $\begin{array}{c}-0,038 \\
(0,034)\end{array}$ & $\begin{array}{c}0,047 * \\
(0,027)\end{array}$ & $\begin{array}{c}0,022 \\
(0,031)\end{array}$ & $\begin{array}{c}0,079 * * * \\
(0,025)\end{array}$ & $\begin{array}{c}0,017 \\
(0,034)\end{array}$ \\
\hline Deportes & $\begin{array}{c}0,018 \\
(0,018)\end{array}$ & $\begin{array}{c}0,019 \\
(0,018)\end{array}$ & $\begin{array}{c}0,036 * \\
(0,018)\end{array}$ & $\begin{array}{c}-0,025 \\
(0,017)\end{array}$ & $\begin{array}{c}0,025 \\
(0,019) \\
\end{array}$ & $\begin{array}{c}-0,031 * * \\
(0,015)\end{array}$ & $\begin{array}{c}0,037 * * \\
(0,018)\end{array}$ & $\begin{array}{c}0,008 \\
(0,014) \\
\end{array}$ & $\begin{array}{c}0,031 \\
(0,020)\end{array}$ \\
\hline $\mathbf{R}^{2}$ & 6,3 & 8,3 & 3,5 & 3,4 & 4 & 2,6 & 7,5 & 2,3 & 6,2 \\
\hline $\mathbf{F}$ & $7,236 * * *$ & $9,624 * * *$ & $3,872 * * *$ & $3,772 * * *$ & $4,489 * * *$ & $2,880 * * *$ & $8,662 * * *$ & $2,592 * * *$ & $7,107 * * *$ \\
\hline
\end{tabular}

$* * * \mathrm{p}<0,01, * * \mathrm{p}<0,05, * \mathrm{p}<0,10$ 


\section{Conclusiones}

Los numerosos estudios realizados acerca de las actitudes hacia la publicidad han tratado de identificar las variables que influyen en que esta actitud sea positiva o negativa. Dado que la actitud que el consumidor tiene hacia la publicidad determina su posterior comportamiento de compra, es muy útil para la empresa que desea vender sus productos optimizar sus impactos publicitarios y dirigirlos en mayor medida a aquellos que pueden más receptivos con el mensaje. La literatura ha llegado a la conclusión de que las caracteristicas personales, esto es, el tipo de personalidad que uno tiene explica el modo en que se comporta y la manera en que recibe el mensaje comercial. También se han encontrado evidencias de que el tipo de contenidos de televisión que se prefieren predicen la forma más o menos activa de ver la televisión. (Hawkins et al., 2001). El objetivo de este trabajo ha sido analizar la influencia de los géneros televisivos preferidos sobre las actitudes hacia la publicidad. Para conseguirlo se han empleado datos procedentes de una encuesta original a telespectadores españoles en los que se pregunta tanto por preferencia de géneros televisivos como por actitudes hacia la publicidad y por los atributos que se encuentran en ella.

Los resultados presentados y analizados en este trabajo apuntan a que los espectadores que muestran una mayor preferencia por los programas de entretenimiento son más susceptibles de tener una percepción más favorable de la publicidad en televisión, tanto en términos generales como para varias de las dimensiones que la componen. De esta manera, valoran más la publicidad como fuente de información de los productos, encuentran que la publicidad les da pistas para la construcción de su propia imagen social, experimentan disfrute en el visionado de los anuncios, y la consideran beneficiosa para la economía, en el sentido de que la potencia y contribuye al crecimiento enconómico y por tanto a un mayor bienestar. Los efectos negativos tradicionalmente asociados a la publicidad, como impulsora del materialismo y corruptora de valores no son compartidos por los aficionados a los programas de entretenimiento. Simultáneamente, los telespectadores a los que les gustan los programas de entretenimiento tienden a no considerar la publicidad como intrusiva ya que los anuncios son percibidos como una continuación del propio programa. Como resultado final, su actitud general hacia la publicidad es favorable. Ello se ajusta a nuestras expectativas iniciales, y una explicación para estas actitudes, que no son compartidas con los que muestran preferencia por otro tipo de programas es que los programas de entretenimiento tienen un formato que se asemeja en mayor medida al formato de la publicidad. Además los aspectos de la personalidad que influyen en la preferencia por unos géneros televisivos sobre otros serían coincidentes con el tipo de personalidad que explicaría el modo en que uno se comporta ante la televisión y la manera en que recibe el mensaje comercial.

Estos resultados sugieren que la inversión publicitaria sería más efectiva para los anunciantes, al menos en términos de atención del telespectador, si los anuncios se ubican 
durante los programas de entretenimiento, al ser los espectadores de este género los que más favorables se muestran hacia la publicidad, En consecuencia, insertar anuncios durante los programas de entretenimiento puede constituir una estrategia efectiva para los anunciantes, ya que a diferencia de lo ocurre con los espectadores que prefieren otros géneros como los informativos o, en menor medida, la ficción, aquellos a los que les gusta el entretenimiento los anuncios no les molestan y su actitud hacia la publicidad es favorable. Esto redundaría en una mayor predisposición hacia la compra de los productos y servicios ofrecidos rentibilizando en mayor media la inversión en publicidad de los anunciantes.

\section{REFERENCIAS BIBLIOGRÁFICAS}

AltMAN, R. (2000): Los géneros cinematográficos. Paidós, Barcelona.

AlwitT, L., PrabHaker, P. (1994): "Identifying Who Dislikes Television Advertising: Not by Demographics Alone", en Journal of Advertising Research 34(6), pp. 17-29.

Andrews, J., Durvasula, S., Netemeyer, R. (1994): "Testing the Cross-National Applicability of U.S. and Russian Advertising Belief and Attitudes Measures", en Journal of Advertising, 23(1) 1, pp. 71-82.

Aumont, J. Marie, M. (2006): Diccionario teórico y crítico del cine. La marca. Buenos Aires, Argentina.

BAGOZZI, R P. (1984): Advanced Methods of Marketing Research, Blackwell, Oxford, Inglaterra.

Bauer, R. A. Greyser, S. A. (1968): Advertising in America: The Consumer View. Boston Division of Research, Graduate School of Business Administration, Harvard University.

BRETT, A.S. M., GNOTH, J., StRong, C., (2009): "Temporal construal in advertising", en Journal of Advertising, 38(3), pp.5-19.

ChAN, K., Li, L., DieHL, S., TerlutTer, R. (2007): "Consumers' Response to Offensive Advertising: A Cross Cultural Study", en International Marketing Review, 24(5), pp. 606-28.

DIX, S., PHAU, I. (2010): "Measuring situational triggers of television channel switching", en Marketing Intelligence and Planning, 28, pp. 137-150.

DUtTA-BERgMAN, M.J. (2006): "The demographic and psychographic antecedents of attitude towards advertising", en Journal of Advertising Research, 46, pp.102-112.

Durvasula, S., Andrews, J.C., Lyonski, S, Netemeyer R. G. (1993): "Assessing the Cross-National Applicability of Consumer Behaviour Models", en Journal of Consumer Research, 19(4), pp. 626-36. 
Gauzente, C. (2008): "Attitudes toward advertising, perceived intrusiveness, perceived ad-clutter and behavioural consequences: A preliminary study" en Proceedings-International Workshop on database and Expert Systems Applications, DEXA; art. N04624760, pp. 461-465.

Hawkings, R.P., Pingree, S., Hitchon, J., Gorham, B. W., Kannaovakun, P., Gilligan, E., Radler, B., Kolbeins, G.H., Schmidt, T. (2001): "Predicting Selection and Activity in Television Genre Viewing", en Media Psychology, 3 (3), pp.237263.

Hellman, H. (2001): "Diversity-An End in Itself. Developing a multi-measure methodology of television programme variety studies", en European Journal of Communication, 16, pp.181-208.

Judge, G. G., Hill, R. C., Griffiths, W. E., LütKePohl, H., Lee, T-C, (1988): Introduction to the Theory and Practice of Econometrics. John Wiley \& Sons, Nueva York, Estados Unidos.

Kantar Media (2013).: Anuario de Audiencia de televisión 2013.

LACEY, N. (2000): Narrative and genre. Key concepts in media studies, Macmillan Press, London.

MACKenZIE, S.B., LUTZ, R.J. (1989): "An empirical examination of the structure antecedents or attitude toward the ad in an advertising pretesting context", en Journal of Marketing, 53, pp. 48-65.

Martin-Santana, J. D., Beerli-Palacio, A. (2008): "Why Attitudes towards Advertising are not Universal: Cultural Explanations", en Journal of Euromarketing, 17(3-4), pp. 159-81.

MehtA, A. (2000): "Advertising Attitudes and Advertising Effectiveness", en Journal of Advertising Research, 40(3), pp. 67-72

NAN, X. (2006): "Perceptual Predictors of Global Attitude toward Advertising: An Investigation of Both Generalized and Personalized Beliefs", en Journal of Current Issues and Research in Advertising, 28(1), pp. 31-44.

NEALE, S. (1980): Genre. British Film Institute, London.

NORRIS, V. P. (1984): "The Economics Effects of Advertising: A Review of the Literature", en Current Issues and Research in Advertising, 7(2), pp. 39134.

Nunnally, J. (1978): Psychometric methods. McGraw-Hill, New York, NY

Petrovici, D., Marinov, M. (2007): "Determinants and antecedents on general attitudes towards advertising. A study of two accession countries", en European Journal of Marketing, 41(3/4), pp. 307-326. 
Podsakoff, N. P., Shen, W.,Podsakoff, P. M. (2006): "The Role of Formative Measurement Models in Strategic Management Research: Review, Critique, and Implications for Future Research", en Research Methodology in Strategy and Management, 3(1), pp.197-252.

Pollay, R., MitTAL, B. (1993): "Here's the Beef: Factors, Determinants, and Segments in Consumer Criticism of Advertising", en Journal of Marketing, 57(3), pp. 99-114.

Ramaprasad, J., M.L. Thurwanger, (1998): "South Asian Students Attitudes toward and Beliefs about Advertising: Measuring across Cultures", en International Communication Division, AEJMC Annual Conference, Baltimore, MD.

RICHINS, M. (1991): "Social Comparison and the Idealized Images of Advertising", en Journal of Consumer Research, 18(1) pp. 71-83.

Roberts, J. A., MANOlis, C. (2000): "Baby boomers and busters: an exploratory investigation of attitudes toward marketing, advertising and consumerism", en Journal of Consumer Marketing, 17(6), pp. 481-497.

RojAS-MÉndez, J.I., DAVIES, G., MAdran. C. (2009): "Universal Differences in Advertising Avoidance Behaviour: A Cross-Cultural Study", en Journal of Business Research, 62(10), pp. 947-954.

SÁnChez-Revilla, M. A. (2013): Estudio Infoadex de la Inversión Publicitaria 2013. Infoadex.

ShavitT, S., Lowery, P., Haefner, J. (1998): "Public Attitudes toward Advertising: More Favourable than you Might Think", en Journal of Advertising Research, 38(4), pp.7-22.

Shim, J. W., PAUL B. (2007): "Effects of Personality Types on the Use of Television Genre", en Journal of Broadcasting \& Electronic Media, 51(2), pp. 287-304.

TSE, A.C.B., LeE. R.P.W. (2001): "Zapping Behaviour during Commercial Breaks" en Journal of Advertising Research, 41(3), pp. 25-29.

VAN MeURS, L. (1998): "Zapp! A study on switching behaviour during commercial breaks"; en Journal of Advertising Research, 38(1), pp. 43-53.

Wollin, L. D. (2002): "Beliefs, Attitudes and Behaviour towards Web Advertising", en International Journal of Advertising, 21(1), pp. 87-113.

[Recibido: 28 de noviembre de 2013. Aceptado con cambios: 20 de octubre de 2014. Aceptación definitiva: 28 de noviembre de 2014]. 